\title{
Nutritional Support of Very Low Birth Weight Infants in a Tertiary Center in a Developing Country
}

\author{
Manar Al-lawama ${ }^{\mathrm{a}, \mathrm{c}}$, Haneen Abu Alrous ${ }^{\mathrm{a}}$, Haitham Alkhatib ${ }^{\mathrm{a}}$, Abdelkareem Alrafaeh ${ }^{\mathrm{a}}$, \\ Zaid Wakileha, Bushra Alawaisheh ${ }^{a}$, Aseel Saadeh ${ }^{a}$, Jumana Sharab ${ }^{\text {, }}$ \\ Eman Badran ${ }^{\mathrm{a}}$, Abla Albsoul-Younes ${ }^{\mathrm{b}}$
}

\begin{abstract}
Background: Very low birth weight infants (VLBWIs) are at high risk for nutritional deficiency. Enteral feeding is usually challenged by increased risk of necrotizing enterocolitis (NEC). The nutritional needs of VLBWIs are usually dependent on parenteral nutrition during early postnatal life. This study aimed to evaluate the nutritional service of VLBWIs at Jordan University Hospital.
\end{abstract}

Methods: This was a prospective follow-up study of VLBWIs with birth weight $\leq 1,500 \mathrm{~g}$. Data were extracted from medical charts and laboratory database.

Results: In total, 43 VLBWIs met our inclusion criteria; of them, $21 \%$ were extremely low birth weight infants (ELBWIs). The mean gestational age was 29 weeks, and the mean birth weight was 1,218 g. The mean age of starting feeds was 3 days. Mean full feed age is 2 weeks. The most common side effect of total parenteral nutrition (TPN) was hypertriglyceridemia (35\%).

Conclusions: Nutritional care of VLBWIs is well established in our center. Initiating fortification earlier and working to increase mother's own breast milk supply is vital to improve growth in low resource setting.

Keywords: VLBWI; Enteral nutrition; TPN; Developing country

\section{Introduction}

Very low birth weight infants (VLBWIs) are at high risk for nutritional deficiency [1]. Enteral feeding is usually challenged by

Manuscript submitted February 25, 2019, accepted March 7, 2019

aDepartment of Pediatrics, School of Medicine, Jordan University Hospital, University of Jordan, Amman, Jordan

${ }^{b}$ School of Pharmacy, University of Jordan, Amman, Jordan

${ }^{\mathrm{c} C}$ Corresponding Author: Manar Al-lawama, Department of Pediatrics, School of Medicine, Jordan University Hospital, University of Jordan, Amman, Jordan.Email:m.allawama@ju.edu.jo

doi: https://doi.org/10.14740/jocmr3797 increased risk of necrotizing enterocolitis (NEC) [2]. Adopting enteral feeding protocols and encouraging mothers to provide breast milk all decrease the rate of NEC and improve the outcome of very low birth weight premature infants [3].

The nutritional needs of VLBWIs are usually dependent on parenteral nutrition during early postnatal life [4]. Early adequate parenteral nutrition minimizes weight loss, positively affects post-natal growth [5], improves neurodevelopmental outcome [1], and reduces the risk of mortality [4]. However, total parenteral nutrition (TPN) is also associated with complications of sepsis, electrolyte disturbances, nutrient deficiencies, and loss of intravenous access [6].

TPN is challenging in developing countries because of the limited availability of components, shortage of qualified health care professionals, and difficulties in preparation and administration [7]. This prospective study aimed to investigate the nutrition support of VLBWIs to ultimately evaluate current practice and identify areas of improvement. Toward this goal, we documented the nutritional support service of VLBWIs in a level 3 neonatal unit in Amman, Jordan.

\section{Materials and Methods}

This was a prospective study conducted at Jordan University Hospital. This study is part of the project entitled "Using care bundles to improve neonatal mortality and morbidity, The JONeo study".

This study was approved by the deanship of scientific research and by the Review Board of Jordan University Hospital. It was conducted in compliance with the ethical standards of Jordan University Hospital on human subjects as well as with the Helsinki Declaration.

We evaluated all very low birth weight neonates of $\leq 1,500$ $\mathrm{g}$ admitted to the neonatal unit of Jordan University Hospital. The exclusion criteria were death in the first 3 days of life, major congenital anomalies, and hydrops fetalis. Extremely low birth weight infants (ELBWIs) are those with a birth weight $<$ $1,000 \mathrm{~g}$.

\section{TPN service and protocol}

TPN was introduced in our unit in 2003. The preparation was 
performed at the bedside by nurses with aseptic technique. This practice continued until 2014 when a proper neonatal unit-based pharmacy was established.

Currently, TPN solutions are prepared in a specialized pharmacy by a licensed pharmacist. Three rooms are allocated for this service: the preparation room, dispensing room, and an office with a computer with both intra- and internet connections.

The solution is prepared using aseptic technique under laminar flow hood. TPN orders are sent to the pharmacy after morning rounds, and the unit pharmacy operates during day shifts only. Extra bags are stored in a designated refrigerator. During weekends or long holidays, the nurses prepare the TPN solutions.

Extra TPN bags are prepared for night shifts needs. TPN bags are stored in a designated refrigerator in the unit where they are kept for a maximum of $24 \mathrm{~h}$. Amino acid TPN solutions are stable in room temperature for up to $48 \mathrm{~h}$, while lipid TPN solutions are stable at room temperature for $24 \mathrm{~h}$.

Standardized TPN solution is used. The pharmacy prepares an amino acid solution that contains $30 \mathrm{~g} / \mathrm{L}$ amino acids $\left(10 \%\right.$; Aminoven $\left.{ }^{\circledR}\right)$ with a glucose concentration of $10 \%$. TPN is started at a rate of $80 \mathrm{~mL} / \mathrm{kg} /$ day and then increased in a rate of $20 \mathrm{~mL} / \mathrm{kg} /$ day to reach a maximum of $150 \mathrm{~mL} / \mathrm{kg} /$ day. The newborn receives $2.4 \mathrm{~g} / \mathrm{kg}$ of amino acid and a glucose input of $5.5 \mathrm{mg} / \mathrm{kg} / \mathrm{min}$ immediately after birth. Customized solutions are prepared only when there is a medical indication such as hypoglycemia. In such cases, the lipid solution is $20 \%\left(\mathrm{SMOF}^{\circledR}\right)$, and the starting dose is $1 \mathrm{~g} / \mathrm{kg} /$ day, with daily increments of $1 \mathrm{~g} / \mathrm{kg} /$ day until the maximum dose of $3 \mathrm{~g} / \mathrm{kg} /$ day is reached.

Sodium chloride is added in the second day of life and when the serum sodium drops to less than $140 \mathrm{mEq} / \mathrm{dL}$ at a dose of $3 \mathrm{mEq} / 100 \mathrm{~mL}$ of parenteral solution. TPN is gradually tapered as the feeding is advanced.

The Jordan University Hospital neonatal unit protocol requires that all neonates with a birth weight of $\leq 1,500 \mathrm{~g}$ should receive TPN immediately at birth at a rate of $80 \mathrm{~mL} / \mathrm{kg} / \mathrm{day}$. Lipid solution will start on the morning round at the next day. TPN is infused via infusion pumps using a microfilter through a peripheral line, an umbilical venous line, or peripherally inserted catheters.

\section{Enteral feeding}

Feeding is started as soon as breast milk is available. The age at which the infant is weaned off TPN will depend on the breast milk availability and tolerance of enteral feeding. In this study, the age of full feeding was defined as the age when the neonate did not need to revert to TPN due to breast milk unavailability. Gavage feeding is used until the infant reached a body weight of $1,500 \mathrm{~g}$, and post-conceptional age of $\geq 32$ weeks. Feeding on empty breast is allowed earlier when the infant is hemodynamically stable. The mother's own breast milk is used, while premature discharge formula at $22 \mathrm{kcal} / \mathrm{oz}$ is used if breast milk is unavailable.

Vitamin D and iron supplements are started once full feed is reached. Vitamin D is started at 400 - 800 unit/day, while iron is started at $2-3 \mathrm{mg} / \mathrm{kg} /$ day. The iron dose is increased to $6 \mathrm{mg} / \mathrm{kg} /$ day in infants with anemia.

\section{Monitoring}

The serum levels of sodium, potassium and triglycerides of VLBWI on TPN are monitored daily. Their glucose level is also checked routinely $\mathrm{q} 8 \mathrm{~h}$. The progression of lipid infusion depends on the triglyceride level; a $<200 \mathrm{mg} / \mathrm{dL}$ is considered normal. Monitoring is continued until a maximum infusion rate of $150 \mathrm{~mL} / \mathrm{kg}$ is achieved, usually at day 5 of age.

At the age of 1 month, nutritional workup that includes hemoglobin, urea, calcium, phosphorus, and alkaline phosphatase measurements will be performed. Direct bilirubin will be added to the blood work if the neonate is still receiving TPN or has visible jaundice.

Growth parameters are followed regularly for each neonate. Stable neonates are weighed every other day. The body length and head circumference are measured at birth and then followed up on weekly basis. These parameters are plotted on a Fenton chart for premature neonates.

\section{Post-discharge care}

All VLBWIs are followed in our clinic for 2 years for growth and development. Fortification of breast milk was stopped once the infant was discharged, unless there was evidence of extrauterine growth restriction and failure of establishing direct breastfeeding. Post-discharge formula was continued until the first birthday. The post-discharge blood work strategy was individualized according to the infant's status.

\section{Data collection}

Clinical data were prospectively collected by a trained research assistant from medical charts and the laboratory database. VLBWIs were included at birth. Medical charts and the laboratory database were checked.

\section{Statistical analysis}

The means and standard deviations are provided for continuous variables, while frequencies and relative percentages are reported for categorical variables. $\mathrm{P}$ value $<0.05$ was considered significant.

\section{Results}

In total, 43 VLBWIs met our inclusion criteria; of them, $21 \%$ were extremely low birth weight infants (ELBWIs). The mean gestational age was 29 weeks. Ranges of gestational age are presented in Figure 1. Mean birth weight was 1,218 g. The demographic data of the patients are detailed in Table 1. 


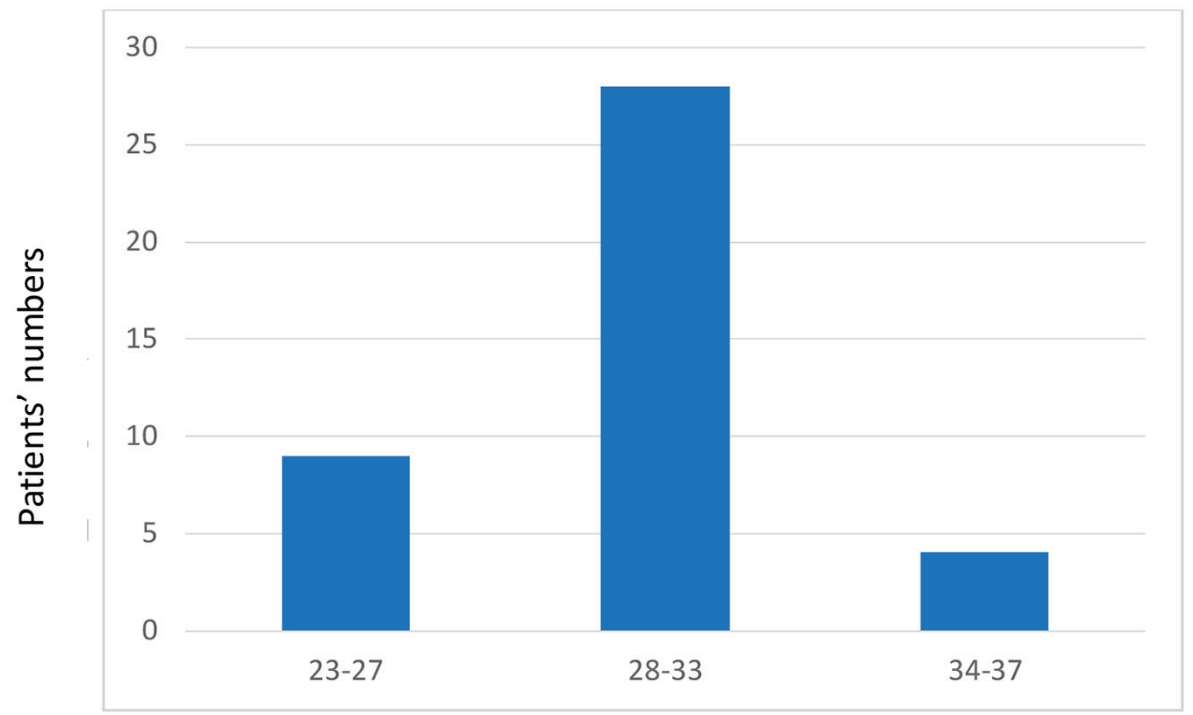

Gestational age ranges

Figure 1. Gestational age ranges among very low birth weight infants included in the study.

The mean age at initiation of feeds was 3 days, and the mean age at full feeding was 15 days. The most common side effect was hypertriglyceridemia (35\%; Table 2$)$. Infants who developed high triglyceride level were those who had lower gestational age $(28 \pm 2.8$ vs. $30 \pm 2.5$ weeks, $\mathrm{P}=0.02$; Table $3)$.

Table 1. Demographic and Clinical Data of Very Low Birth Weight Infants Included in the Study

\begin{tabular}{|ll}
\hline Characteristics & Value \\
\hline Gestational age (week) & $29 \pm 3(23-37)$ \\
\hline $23-27$ & 9 \\
$28-32$ & 28 \\
\hline $33-37$ & 4 \\
\hline Birth weight & $1218 \pm 253(660-1,500)$ \\
\hline Extremely low birth weight infants & $10(23)$ \\
\hline Small for age & $15(35)$ \\
Male gender & $22(51 \%)$ \\
Cesarean section & $33(77 \%)$ \\
\hline Low Apgar score & $5(12 \%)$ \\
Chorioamnionitis & $1(2 \%)$ \\
Prolonged rupture of membranes & $12(28 \%)$ \\
GBS positive & $2(5 \%)$ \\
\hline GBS unknown & $41(95 \%)$ \\
Preeclampsia & $1(2 \%)$ \\
Antenatal steroids & $29(67 \%)$ \\
NEC stage 3 & $1(2 \%)$ \\
Mortality & $6(14 \%)$ \\
\hline
\end{tabular}

\section{Discussion}

The nutrition service for VLBWIs is well established in our center. In our experience, quality care could be achieved even with limited resources through dedication and multidisciplinary team work. The current study cohort included all neonates admitted to our unit with a birth weight of $\leq 1,500 \mathrm{~g}$; of these, $21 \%$ were ELBWIs. The majority were born via cesarean section $(77 \%)$, and only two-thirds of them received antenatal steroids (Table 1).

Our enteral feeding protocol depends on the early initiation of feeding. As shown in our results, the mean age when enteral feeding was initiated was 3 days (Table 2). Early initiation of enteral feeding has been proven to decrease mortality and the rate of NEC $[8,9]$. We adopted a tapered increase protocol of $20 \mathrm{~mL} / \mathrm{kg} / \mathrm{day}$. Although previous reports have shown that this did not decrease the rate of NEC compared to rapid

Table 2. Nutritional Data of Very Low Birth Weight Infants Included in the Study

\begin{tabular}{ll}
\hline Characteristics & Value (frequency) \\
\hline Triglyceride $200-399 \mathrm{mg} / \mathrm{dL}$ & $9(21 \%)$ \\
Triglyceride $>400 \mathrm{mg} / \mathrm{dL}$ & $5(12 \%)$ \\
Age feeds started, mean (range) & $3(1-6)$ days \\
Age at full feeds (mean) & 15 days \\
Breast milk only & $39(90 \%)$ \\
Hypophosphatemia & $2 / 28(7)$ \\
Elevated alkaline phosphatase & $9 / 27(33)$ \\
Blood transfusion & $18(42 \%)$ \\
Extrauterine growth restriction & $30(70)$ \\
\hline
\end{tabular}


Table 3. Comparison Between Very Low Birth Weight Infants With Hypertriglyceridemia and Those With Normal Triglyceride

\begin{tabular}{llll} 
& Hypertriglyceridemia (14) & Normal triglyceride (29) & P value \\
\hline Birth weight & $1,146 \pm 291$ & $1,273 \pm 211$ & 0.1 \\
Extremely low birth weight & $6(42 \%)$ & $4(13.7)$ & 0.4 \\
Gestational age & $28 \pm 2.8$ & $30 \pm 2.5$ & $0.02 *$ \\
\hline
\end{tabular}

*Significant $P$ value.

increase protocols $(25-40 \mathrm{~mL} / \mathrm{kg} /$ day) [10], the rate of $\mathrm{NEC}$ is low [11]; thus, changing our protocol to an increment rate of $25 \mathrm{~mL} / \mathrm{kg} /$ day is safe and would decrease the number of TPN days by 1 day. This would help in decreasing the number of central line days and corresponding blood stream infection [12] and TPN complications [6].

The availability of breast milk is another limiting factor in advancing enteral feeds. The rate of mothers who had breast milk is higher at $90 \%$ (Table 2) in this cohort than the $15 \%$ in our previous data [11]. This might reflect improvement in the competency of the unit staff in counseling mothers; however, it may also be an overestimation of the actual rate due to the shorter duration of the current study.

Despite our early initiation of feeding, the average age of reaching full feeding was 15 days (Table 2), and this is similar to a previous finding [13]. Premature infants weighing 1,000 - 1,500 $\mathrm{g}$ at birth are expected to achieve full feeding earlier [14]. In our unit, many mothers of premature infants have difficulty in having adequate milk supply, and thus their infants will need to go back on TPN until a sufficient amount of milk is provided. Hence, although their infants reached full feeding earlier than the defined age of full feeding, their recurrent need for TPN increased the full feeding age in this study. Our hospital has recently started to apply for accreditation as a baby-friendly hospital. This opportunity will ensure better competency for our neonatal nurses, and it will drive technical support from national and international agencies that will help in increasing the efficacy of breast bumping [15].

Breast milk alone is insufficient to support the growth of premature VLBWIs [14], but fortification improves their growth [16]. However, human milk fortifiers were not approved by the Jordan Food and Drug Administration; instead, breast milk was fortified using premature discharge formula, a practice documented before in our region [17]. Fortification was started once the infants reached full feeds. Fortification as early as the infant reaches $20 \mathrm{~mL} / \mathrm{kg}$ /day of feeding was shown to be safe [18], and starting fortification earlier than our current protocol might help to improve growth.

The process of prescription, preparation and administration of TPN is well established. Despite the lack of intravenous multivitamins and trace elements, amino acid and lipid solutions are also fundamental in parenteral nutrition. The shortage in TPN solution is becoming a worldwide issue [19]. Early initiation and more rapid advancement of enteral feeding should help to overcome this issue.

Our current TPN protocol is standardized TPN. Standardized TPN provides the same nutritional value as customized TPN evidenced by the comparable body weight gain [20] and similar biochemical levels in premature infants, but at a $30 \%$ lower cost [21]. Moreover, it is believed to decrease the prescription time and prescription errors [22].

Our current TPN solution provides a starting protein intake of $2.4 \mathrm{~g} / \mathrm{kg} /$ day at a rate of $80 \mathrm{~mL} / \mathrm{kg} /$ day starting from birth, with a maximum of $4.5 \mathrm{~g} / \mathrm{kg} /$ day. Because our dosage is within the recommended levels [23], the urea level is not evaluated to monitor the side effects of the amino acid solution. The TPN is $10 \%$ dextrose and gives a glucose input of $5.6 \mathrm{mg} / \mathrm{kg} / \mathrm{day}$, and the final rate of $150 \mathrm{~mL} / \mathrm{kg} /$ day gives a glucose input of $10 \mathrm{mg} / \mathrm{kg} /$ day. Both rates are within recommended levels [23]. There was no significant hypoglycemia or hypoglycemia reported during the study period.

Lipid metabolism and clearance in very low birth weight premature infants is lower than that in term infants, and the serum triglyceride level is proportionate to the rate of infusion [24]. According to our protocol, lipid solution is started early on day 2 of life with a dose of $1 \mathrm{~g} / \mathrm{kg} /$ day and increments of 1 $\mathrm{g} / \mathrm{kg} /$ day depend on the triglyceride level, and the solution is infused over $24 \mathrm{~h}$. The most common side effect of TPN in our cohort was intolerance to lipid solution, with hypertriglyceridemia documented in $35 \%$ of the cohort. Of these, $12 \%$ had a triglyceride level $>400 \mathrm{mg} / \mathrm{dL}$. Similar to previous report [25], we also found that lower gestational age infants are at higher risk of lipid intolerance (Table 3) [26]. In extremely premature infants born at $\leq 28$ weeks gestational age, starting TPN at 0.5 $\mathrm{g} / \mathrm{kg} /$ day and lowering the increment to $0.5 \mathrm{~g} / \mathrm{dL}$ should help decrease the risk of hypertriglyceridemia.

At a rate of $150 \mathrm{~mL} / \mathrm{kg} /$ day, our TPN solution provides $108 \mathrm{kcal} / \mathrm{kg} /$ day and a protein-to-energy ratio of $4.5 \mathrm{~g} / 100$ $\mathrm{kcal}$. The lipid solution provides $50 \%$ of non-protein energy, which is slightly higher than the recommended ratio of $25-$ $40 \%$ [26].

In enterally fed VLBWIs, enteral and parenteral feeding overlaps, and thus the presented doses and ratios are factual only for those who are not receiving enteral feeding.

TPN cholestasis is a known side effect of prolonged TPN [25]. In this study, this was defined as a direct bilirubin of $>2$ $\mathrm{mg} / \mathrm{dL}$, but no such cases were observed. This may be due to our feeding protocol, the exclusion of infants who underwent surgery, and the use of SMOF lipid solution [27].

Our TPN solution lacks calcium and phosphate supplements as they are not available in Jordan. Moreover, our current feeding protocol does not provide enteral calcium or phosphate supplements. Metabolic bone disease is another complication of prematurity [28]. In our protocol, serum phosphate and alkaline phosphatase levels at 1 month of age is used to identify this complication. Alkaline phosphatase is considered abnormal when its level is $>500 \mathrm{IU} / \mathrm{dL}$, and this was found in $33 \%$ of our cohort. Two premature infants $(7 \%)$ had 
hypophosphatemia (i.e. phosphate level $<2.0 \mathrm{mmol} / \mathrm{dL}$ ), and one of them did not show elevated alkaline phosphatase (Table 2 ). Using radiography to evaluate for bone mineralization is not utilized in our unit.

Elevated alkaline phosphatase level is a reliable indicator of bone demineralization in premature infants; however, normal levels also do not guarantee normal bones [29]. Our current protocol of combined measurement of both alkaline phosphates and phosphate has $100 \%$ sensitivity rate [30]. However, cases of metabolic bone disease might be missed. Radiography should be additionally performed to evaluate metabolic bone disease, particularly in ELBWIs as they are at the highest risk of such condition, to increase the detection rate.

Oral vitamin D supplements at a dose of 400 - 800 units are started once the newborn reaches full enteral feeding. Starting vitamin D supplement earlier with a fixed dose of 1,000 units [31] in addition to early fortification of breast milk might help to improve the rate of metabolic bone disease. Extrauterine growth restriction is prevalent in VLBWIs [32, 33], and 70\% of infants in the current study had the condition.

In conclusion, nutritional support of VLBWIs in our center is well established. Increasing the advancement rate of enteral feeding, starting early fortification, and improving vitamin D and calcium phosphate supplements should be adopted to improve the nutritional status of VLWBIs. Clinical studies of these strategies are needed.

\section{Acknowledgments}

We thank Miss Wafa Rabayaa for her efforts in data collection.

\section{Funding}

This study was funded by the deanship of scientific research at the University of Jordan and by the scientific research fund.

\section{Conflict of Interest}

The authors have no conflict of interest to declare.

\section{Informed Consent}

This was a prospective follow-up of the outcomes of VLBWIs receiving the standard of care. Data were extracted on daily basis from medical charts and laboratory database. Informed consent is not needed.

\section{Author Contributions}

Manar Al-lawama: idea, design, analysis, and drafting the manuscript. Haneen Abu Alrous, Haitham Alkhatib, Abdelkareem Alrafaeh, Zaid Wakileh, Bushra Alawaisheh, Aseel Saadeh, and Jumana Sharab: gathering data, drafting manuscript, and approving manuscript. Eman Badran: acquisition of data, and approving the manuscript. Abla Albsoul-Younes: drafting the manuscript, and approving the manuscript.

\section{References}

1. De Curtis M, Dito L, Lucchini R, Terrin G. Nutrition of very low birth-weight infants. Ital J Pediatr. 2014;40(Suppl 1):A39. S1-A39.

2. Rich BS, Dolgin SE. Necrotizing enterocolitis. Pediatr Rev. 2017;38(12):552-559.

3. Patel AL, Panagos PG, Silvestri JM. Reducing incidence of necrotizing enterocolitis. Clin Perinatol. 2017;44(3):683-700.

4. Parish A, Bhatia J. Early aggressive nutrition for the premature infant. Neonatology. 2008;94(3):211-214.

5. Meyers JM, Greecher CP, Shaffer ML, Shenberger JS. Potential influence of total parenteral nutrition on body composition at discharge in preterm infants. J Matern Fetal Neonatal Med. 2013;26(15):1548-1553.

6. Zingg W, Tomaske M, Martin M. Risk of parenteral nutrition in neonates - an overview. Nutrients. 2012;4(10):1490-1503.

7. Murguia-Peniche T, Kirsten GF. Meeting the challenge of providing neonatal nutritional care to very or extremely low birth weight infants in low-resource settings. World Rev Nutr Diet. 2014;110:278-296.

8. Hamilton E, Massey C, Ross J, Taylor S. Early enteral feeding in very low birth weight infants. Early Hum Dev. 2014;90(5):227-230.

9. Sanchez-Tamayo T, Espinosa Fernandez MG, Affumicato L, Gonzalez Lopez M, Fernandez Romero V, Moreno Algarra MC, Salguero Garcia E. [Reduction in necrotising enterocolitis after implementing an evidence-based enteral nutrition protocol in very low birth weight newborns]. An Pediatr (Barc). 2016;85(6):291-299.

10. Oddie SJ, Young L, McGuire W. Slow advancement of enteral feed volumes to prevent necrotising enterocolitis in very low birth weight infants. Cochrane Database Syst Rev. 2017;8:CD001241.

11. Al-lawama M, Abdelghani T, Badran E. Mortality and short-term outcomes of very low birth weight infants at a tertiary care center in Jordan: comparison with other countries. Pediatr Ther. 2017;7:311.

12. Al Lawati TT, Al Jamie A, Al Mufarraji N. Central line associated sepsis in children receiving parenteral nutrition in Oman. J Infect Public Health. 2017;10(6):829832.

13. Dutta S, Singh B, Chessell L, Wilson J, Janes M, McDonald $\mathrm{K}$, Shahid S, et al. Guidelines for feeding very low birth weight infants. Nutrients. 2015;7(1):423-442.

14. Mangili G, Garzoli E. Feeding of preterm infants and fortification of breast milk. Pediatr Med Chir. 2017;39(2):158.

15. El Sakka A, El Shimil MS, Ibrahium S. Effect of implementing breastfeeding promotion program in neonatal intensive care unit on newborn weight and breastfeeding practices. J Matern Fetal Neonatal Med. 2018;28:1-171.

16. Gupta V, Rebekah G, Sudhakar Y, Santhanam S, Kumar 
M, Thomas N. A randomized controlled trial comparing the effect of fortification of human milk with an infant formula powder versus unfortified human milk on the growth of preterm very low birth weight infants. J Perinat Med. 2017;45(8):953-957.

17. El Sakka A, El Shimi MS, Salama K, Fayez H. Post discharge formula fortification of maternal human milk of very low birth weight preterm infants: an introduction of a feeding protocol in a university hospital. Pediatr Rep. 2016;8(3):6632.

18. Alizadeh Taheri P, Sajjadian N, Asgharyan Fargi M, Shariat M. Is early breast milk fortification more effective in preterm infants? A clinical trial. Nutr Clin Pract. 2016;31(4):556-559.

19. Plogsted S, Adams SC, Allen K, Cober MP, Greaves J, Mogensen KM, Ralph A, et al. Parenteral nutrition multivitamin product shortage considerations. Nutr Clin Pract. 2016;31(4):556-559.

20. Evering VH, Andriessen P, Duijsters CE, Brogtrop J, Derijks LJ. The effect of individualized versus standardized parenteral nutrition on body weight in very preterm infants. J Clin Med Res. 2017;9(4):339-344.

21. Yeung MY, Smyth JP, Maheshwari R, Shah S. Evaluation of standardized versus individualized total parenteral nutrition regime for neonates less than 33 weeks gestation. J Paediatr Child Health. 2003;39(8):613-617.

22. Cerra FB. A standardized TPN order form reduces staff time and potential for error. Nutrition. 1990;6(6):498499.

23. Working Group of Pediatrics Chinese Society of Parenteral, Enteral Nutrition, Working Group of Neonatology Chinese Society of Pediatrics, Working Group of Neonatal Surgery Chinese Society of Pediatric Surgery.
CSPEN guidelines for nutrition support in neonates. Asia Pac J Clin Nutr. 2013;22(4):655-663.

24. Putet G. Lipid metabolism of the micropremie. Clin Perinatol. 2000;27(1):57-69, v-vi.

25. Jolin-Dahel K, Ferretti E, Montiveros C, Grenon R, Barrowman N, Jimenez-Rivera C. Parenteral nutrition-induced cholestasis in neonates: where does the problem lie? Gastroenterol Res Pract. 2013;2013:163632.

26. Holtrop P, Swails T, Riggs T. Hypertriglyceridemia in extremely low birth weight infants receiving lipid emulsions. J Neonatal Perinatal Med. 2015;8(2):133-136.

27. Deshpande G, Simmer K, Deshmukh M, Mori TA, Croft KD, Kristensen J. Fish Oil (SMOFlipid) and olive oil lipid (Clinoleic) in very preterm neonates. J Pediatr Gastroenterol Nutr. 2014;58(2):177-182.

28. Rehman MU, Narchi H. Metabolic bone disease in the preterm infant: Current state and future directions. World J Methodol. 2015;5(3):115-121.

29. Abdallah EA, Said RN, Mosallam DS, Moawad EM, Kamal NM, Fathallah MG. Serial serum alkaline phosphatase as an early biomarker for osteopenia of prematurity. Medicine (Baltimore). 2016;95(37):e4837.

30. Tinnion RJ, Embleton ND. How to use... alkaline phosphatase in neonatology. Arch Dis Child Educ Pract Ed. 2012;97(4):157-163.

31. Mathur NB, Saini A, Mishra TK. Assessment of adequacy of supplementation of vitamin d in very low birth weight preterm neonates: a randomized controlled trial. J Trop Pediatr. 2016;62(6):429-435.

32. Ehrenkranz RA. Extrauterine growth restriction: is it preventable? J Pediatr (Rio J). 2014;90(1):1-3.

33. Cole TJ, Statnikov Y, Santhakumaran S, et al. Arch Dis Child Fetal Neonatal Ed. 2014;99:F34-F40. 